\title{
Paratiroidectomía por hiperparatiroidismo en una serie de pacientes mexicanos: características clínicas y demográficas, nuestra experiencia
}

\author{
Parathyroidectomy for hyperparathyroidism in a serie of Mexican \\ patients: clinical and demographic characteristics, our experience
}

\author{
Estefanía Boyer Duck, ${ }^{*} \neq$ Pablo Cortés Romano, ${ }^{\ddagger}$ Adriana Rodríguez Gómez, ${ }^{\S}$ Fernando Espinosa Lira*,‡ \\ Citar como: Boyer DE, Cortés RP, Rodríguez GA, Espinosa LF. Paratiroidectomía por hiperparatiroidismo en una serie de pacientes mexicanos: \\ características clínicas y demográficas, nuestra experiencia. Acta Med. 2021; 19 (1): 40-44. https://dx.doi.org/10.35366/98568
}

\section{Resumen}

Introducción: El hiperparatiroidismo primario (HPP) es la tercera alteración endócrina más frecuente en el mundo, con una prevalencia estimada de $1 \%$ en mayores de 65 años. Caracterizada por presencia de niveles elevados de calcio y de paratohormona (PTH). El objetivo de este estudio retrospectivo es describir características clínicas y demográficas de casos con HPP sometidos a paratiroidectomía en hospital privado de tercer nivel en la Ciudad de México. Material y métodos: Estudio retrospectivo de una serie de pacientes con HPP sometidos a paratiroidectomía, por diferentes cirujanos, periodo de enero de 2015 a diciembre de 2018. Incluyendo 31 pacientes, se obtuvo la base del Servicio de Patología y posteriormente se buscaron los expedientes. Se buscaron datos clínicos, demográficos, laboratorio y de cirugía. Resultados: La media de edad de población estudiada fue de 56.48 años ( $D E \pm 13.7) ; 24$ (77.41\%) fueron mujeres. Causa principal fue adenoma. En el análisis multivariado de regresión logística, los factores de riesgo independientes para duración de cirugía mayor a 60 minutos fueron hipertensión y dislipidemia, todos los pacientes consiguieron normocalcemia, sin existir complicaciones transquirúrgicas o postquirúrgicas. Conclusiones: La hipertensión y dislipidemia se identificaron como factores de riesgo independientes para mayor duración (> 60 minutos) de paratiroidectomía. La paratiroidectomía es curativa en manos expertas.

Palabras clave: Hiperparatiroidismo primario, paratiroidectomía, hipercalcemia, hipertensión, dislipidemia.

\section{Abstract}

Introduction: Primary hyperparathyroidism $(\mathrm{PPH})$ is the third most frequent endocrine disorder in the world with an estimated prevalence of $1 \%$ in people over 65 years. It is characterized by the presence of elevated calcium levels and elevated levels of paratohormone (PTH). The objective of this retrospective study is to describe clinical and demographic characteristics of patients with $\mathrm{PPH}$, who had parathyroidectomy in a third level private hospital in Mexico City. Material and methods: Retrospective study of a group of patients diagnosed with PPH who had parathyroidectomy, by different surgeons, period from January 2015 to December 2018. We included 31 patients of which the data was obtained from pathology service; clinical, demographic, laboratory and surgery data were recorded. Results: The average age of the study population was 56.48 years (SD \pm 13.7$), 24$ (77.41\%) were women. Main cause was adenoma. In the multivariate logistic regression analysis, the independent risk factors for the duration of surgery greater than 60 minutes were hypertension and dyslipidemia, all patients achieved normocalcemia, without there being any trans-surgical or post-surgical complications. Conclusions: Hypertension and dyslipidemia are identified as independent risk factors for longer duration (> 60 minutes) for parathyroidectomy. Parathyroidectomy is a curative procedure in expert hands.

Keywords: Primary hyperparathyroidism, parathyroidectomy, hypercalcemia, hypertension, dyslipidemia.
* Facultad Mexicana de Medicina de la Universidad La Salle. Ciudad de México, México.

‡ Departamento de Cirugía General del Hospital Ángeles Pedregal. Ciudad de México, México.

$\S$ Departamento de Patología del Hospital Ángeles Pedregal. Ciudad de México, México.
Correspondencia:

Estefanía Boyer Duck

Correo electrónico: estefania_boyerd@hotmail.com

Aceptado: 12-05-2020.

www.medigraphic.com/actamedica 


\section{INTRODUCCIÓN}

El hiperparatiroidismo primario (HPP) es la tercera alteración endócrina más frecuente en el mundo con una prevalencia estimada de $1 \%$ en mayores de 65 años. Se caracteriza por la presencia de niveles elevados de calcio y niveles elevados de paratohormona (PTH). Afecta principalmente a mujeres con una proporción de 3.4:1.1,2

Entre las causas más frecuentes de HPP se encuentran: adenoma único en 75-85\%, hiperplasia de las glándulas paratiroides (dos glándulas 2-12\%, tres glándulas 1-2\%, cuatro o más glándulas $<1-15 \%$ ), carcinoma de paratiroides $1 \%{ }^{2}$

En alrededor del 80 a 85\% de los casos, el hiperparatiroidismo primario es asintomático, sin embargo, su incidencia ha ido en aumento gracias a que en las últimas décadas se realiza con mayor frecuencia el tamizaje de niveles de calcio sérico en los pacientes. ${ }^{3,4}$

La cirugía continúa siendo el tratamiento de elección para el HPP, por lo que la identificación de la(s) glándula(s) afectada(s) por imagen mediante el uso de tomografía 4D, tecnecio 99-MIBI y/o ultrasonido resultan esenciales para poder realizar una paratiroidectomía a través de mínima invasión. El porcentaje de curación es del $98 \%$ en manos expertas. ${ }^{4,5}$

El objetivo de este estudio retrospectivo es describir las características clínicas y demográficas de pacientes con HPP sometidos a paratiroidectomía en un hospital privado de tercer nivel en la Ciudad de México.

\section{MATERIAL Y MÉTODOS}

Se realizó un estudio retrospectivo de una cohorte de casos con diagnóstico de HPP que fueron sometidos a paratiroidectomía por diferentes cirujanos, en el periodo comprendido de enero de 2015 a diciembre de 2018.

Tabla 1: Características demográficas y clínicas.*

$n(\%)$

Edad, media $\pm \mathrm{DE}$

Género femenino

Hipertensión arterial

Diabetes mellitus II

Cardiopatía isquémica

Dislipidemia

Peso, mediana (IQR)

Duración de cirugía, mediana \pm IQR

$\mathrm{DE}$ = desviación estándar; IQR = rango intercuartil.

* Prueba de normalidad Shapiro-Wilk.
Tabla 2: Frecuencia de glándula afectada.

$\begin{array}{lc}\text { Glándula afectada } & \mathrm{n}(\%) \\ \text { Superior derecha } & 4(12.9) \\ \text { Superior izquierda } & 7(22.5) \\ \text { Inferior derecha } & 5(16.12) \\ \text { Inferior izquierda } & 13(41.9)\end{array}$

El diagnóstico de HPP se estableció con la presencia de niveles elevados de calcio (valor $>10.2 \mathrm{mg} / \mathrm{dL}$ ) y niveles elevados PTH (PTH > 90 pg/mL). Se realizó una búsqueda en la base de datos del Departamento de Patología del hospital. Se encontraron 62 pacientes, de los cuales sólo fue posible obtener 42 expedientes en físico; sin embargo, sólo 31 expedientes se encontraban completos, por lo que el resto fueron excluidos de nuestro estudio. El diagnóstico de hiperparatiroidismo se hizo con base en la PTH y al calcio sérico. El análisis estadístico fue realizado utilizando el programa IBM SPSS para Windows, versión 24. Las variables categóricas fueron expresadas en frecuencias y porcentajes. Las variables continuas como medias y desviación estándar (DE) o medianas con rango intercuartilar (RIQ) de acuerdo con la prueba de normalidad de Shapiro-Wilk. Las variables categóricas fueron comparadas con prueba $\chi^{2}$ o exacta de Fisher. Se realizó análisis multivariado con modelo de regresión logística binaria.

\section{RESULTADOS}

Un total de 31 pacientes fueron incluidos, la media de edad de la población estudiada $(\mathrm{n}=31)$ fue de 56.48 años (DE \pm 13.7$), 24$ (77.41\%) fueron mujeres (Tabla 1). Las comorbilidades más frecuentemente encontradas fueron hipertensión arterial en el 58\%, diabetes mellitus tipo II en el $35.4 \%$, dislipidemia en el $32.2 \%$, y únicamente un paciente había sufrido un infarto agudo al miocardio. Se documentó duración de la cirugía promedio de $62.9 \pm 18.4$ min, con reporte de sangrado mínimo $\left(<10 \mathrm{~cm}^{3}\right)$ en 27 pacientes $(84.3 \%), 10 \mathrm{~cm}^{3}$ en tres $(9.3 \%)$ y $20 \mathrm{~cm}^{3}$ en dos (6.2\%). La glándula más afectada fue la inferior izquierda en $41.9 \%$ de los casos (Tabla 2). Respecto al resultado de patología, $24(77.4 \%)$ pacientes tuvieron adenoma, seis (19.3\%) hiperplasia y uno (3.2\%) microadenoma. Respecto a la cirugía, únicamente se resecó una glándula paratiroides en cada caso, con una media de peso de $4.3 \mathrm{~g}$ ( $\mathrm{DE} \pm 2.4$ ).

Se tomaron estudios de laboratorio prequirúrgicos y postquirúrgicos, los cuales se muestran en las Tablas 3 y 4 . Ninguno de los casos presentó hipocalcemia en el posto- 
peratorio, por lo que no fue necesaria la reposición de calcio; en 26 (81.5\%) la causa de HPP fue por adenoma y en cinco (18.5\%) por hiperplasia paratiroidea confirmado por histopatología en ambos casos.

Todos nuestros pacientes en los laboratorios postquirúrgicos tuvieron normocalcemia.

La hipertensión y dislipidemia se asoció con mayor tiempo de cirugía OR 5.2 ( $\mathrm{p}=0.023$, IC 95\% 3.28-7.1) y OR 6.09 ( $p=0.01$, IC 95\% 4.0-8.1), respectivamente.

\section{DISCUSIÓN}

El HPP es el tipo de hiperparatiroidismo más frecuente, siendo la causa reportada más frecuente el adenoma en $85-90 \%$, en nuestra serie fue de $81.5 \%{ }^{6,7}$ En pacientes asintomáticos, el diagnóstico de HPP suele ser difícil y en la mayoría de las ocasiones el aumento de exámenes de laboratorio de "rutina" han permitido poder diagnosticarlo con mayor frecuencia; sin embargo, se ha estudiado la prevalencia de HPP en diferentes contextos y se ha encontrado hasta en un $11.5 \%$ de casos con índice de masa corporal bajo y densitometría ósea alterada. ${ }^{5}$ En nuestra serie no encontramos algún antecedente relacionado directamente con la presencia de HPP de forma subclínica, aunque es posible que al tratarse de una institución privada los exámenes de "rutina" continúen siendo el común denominador para detectar HPP. En la actualidad, contamos con cirugía de mínima invasión, pero para poder realizarla, es necesario poder tener identificada la(s) glándula(s) que se desea(n) tratar, con imagen preoperatoria. En todos los casos se presentó USG preoperatorio.

Al compararlo con el estudio de Granados y colaboradores $^{8}$ de población mexicana, su media de edad es de 51.8, parecida a la nuestra que es de 56.4 años, la media de calcio es de $11.6 \mathrm{mg} / \mathrm{dL}$ y la nuestra es de $11.1 \mathrm{mg} / \mathrm{dL}$, la principal causa fue por adenoma paratiroideo; sin embargo, en nuestra investigación, al ser un hospital privado, pudimos observar que muchos expedientes no cuentan con todos los estudios de laboratorio o de imagen realizados de forma externa.

Dentro de las principales complicaciones de la cirugía se encuentra la hipocalcemia transitoria, que ocurre en $15-30 \%$ de las personas, aunque en nuestro grupo ninguno presentó hipocalcemia. Si el paciente no desea cirugía o no es candidato por su estado funcional, es necesario dar tratamiento médico para proteger la mineralización ósea. ${ }^{9,10}$

No existe ningún estudio que asocie la hipertensión o dislipidemia con mayor tiempo quirúrgico; con lo que se asocia hipertensión es con la presencia de hiperparatiroidismo primario, y lo relacionan por el efecto hemodinámico que causa el estado de constante hipercalcemia creando y afectando las resistencias vasculares, disfunción y cambios estructurales en las resistencias vasculares. ${ }^{11}$

Algunas limitaciones de nuestro estudio deben ser resaltadas. Se trata de un análisis retrospectivo de una serie de casos que, si bien fueron atendidos en el mismo centro, no cuenta con un servicio exclusivo de cirugía endócrina, lo cual no permite protocolizar a todos del mismo modo. $\mathrm{Al}$ inicio encontramos un número importante de casos de HPP y paratiroidectomía; sin embargo, no todos los expedientes pudieron ser consultados y otros tuvieron que ser descartados por falta de datos, por lo que nuestros resultados no pueden ser extrapolados a la población general, aunque esto abre la posibilidad de realizar un estudio prospectivo que incluya mayor información de los casos y un seguimiento más estricto y a largo plazo.

\section{CONCLUSIONES}

La hipertensión y la dislipidemia se identificaron como factores de riesgo para mayor duración (> 60 minutos) de la paratiroidectomía.

El adenoma es la causa principal de hiperparatiroidismo primario, lo cual concuerda con los resultados de nuestra publicación. Dado que ya se hacen estudios de

\begin{tabular}{|c|c|c|c|}
\hline \multicolumn{4}{|c|}{ Tabla 3: Laboratorios.* } \\
\hline Laboratorios & Prequirúrgico & Postquirúrgico & $p^{* *}$ \\
\hline Calcio, media \pm DE & $11.1 \pm 0.65$ & $9.1 \pm 0.5$ & $<0.00$ \\
\hline Fósforo, media $\pm \mathrm{DE}$ & $2.8 \pm-0.50$ & $3.9 \pm 0.5$ & $<0.00$ \\
\hline Albúmina, mediana (IQR) & $4.1(3.2-4.5)$ & $3.3 \pm 0.45^{\star * *}$ & $<0.00$ \\
\hline PTH, mediana (IQR) & 226.6 (203-312) & $42.4(31.8-43)$ & $<0.00$ \\
\hline
\end{tabular}


Tabla 4: Tabla comparativa de laboratorios prequirúrgicos y postquirúrgicos.

\begin{tabular}{|c|c|c|c|c|c|}
\hline \multicolumn{2}{|c|}{ Calcio } & \multicolumn{2}{|c|}{ Fósforo } & \multicolumn{2}{|c|}{ Paratohormona } \\
\hline Prequirúrgica & Postquirúrgica & Prequirúrgica & Postquirúrgica & Prequirúrgica & Postquirúrgica \\
\hline 9.8 & 9.9 & 4.3 & 4.7 & 201.8 & 59 \\
\hline 11.2 & 9.9 & 2.3 & 3.2 & 317 & 53 \\
\hline 10.9 & 10 & 3.4 & 4 & 255.2 & 22 \\
\hline 11 & 7.8 & 3.1 & 4.6 & 245 & 45 \\
\hline 10 & 8.6 & 3.3 & 3.9 & 125.7 & 12.3 \\
\hline 10.5 & 8.5 & 3.4 & 4.8 & 260.1 & 25 \\
\hline 10.7 & 8.5 & 2.8 & 3.6 & 254 & 26 \\
\hline 11.5 & 8.6 & 2.4 & 3.8 & 192.4 & 32.6 \\
\hline 11.4 & 9.9 & 2.4 & 3.5 & 226.6 & 11.3 \\
\hline 10.9 & 9.5 & 2.8 & 4.09 & 188.9 & 47 \\
\hline 11.6 & 9.8 & 3.1 & 2.9 & 302.9 & 23.8 \\
\hline 11.1 & 9 & 2.6 & 4.16 & 181.9 & 59.2 \\
\hline 11.2 & 9.1 & 2.8 & 4.1 & 177.7 & 38 \\
\hline 11.4 & 8.7 & 3.1 & 3.4 & 295.9 & 53 \\
\hline 11.6 & 9.7 & 2.8 & 3.93 & 413.3 & 58 \\
\hline 12.9 & 9.4 & 2.33 & 3.77 & 431 & 52 \\
\hline 10.3 & 9.6 & 3.05 & 3.16 & 111 & 52.9 \\
\hline 12.5 & 10.1 & 3.21 & 3.3 & 914.8 & 21.4 \\
\hline 10.6 & 9.1 & 3.04 & 4.11 & 64.7 & 23.4 \\
\hline 11.6 & 9.5 & 2.4 & 4.12 & 220.9 & 17.6 \\
\hline 10.2 & 8.4 & 3.11 & 4.2 & 122.4 & 27.9 \\
\hline 10.8 & 9.4 & 2.12 & 4.01 & 368 & 45.4 \\
\hline 11.1 & 8.1 & 2.5 & 4.3 & 171.1 & 49.5 \\
\hline 10.6 & 8.8 & 3.4 & 3.5 & 170.4 & 60.4 \\
\hline 11.6 & 9.3 & 2.5 & 3.9 & 214 & 23.1 \\
\hline 10.8 & 9.5 & 3.8 & 2.6 & 289 & 26 \\
\hline 11.6 & 9.3 & 3.4 & 3.5 & 285.6 & 19.5 \\
\hline 11.5 & 9.3 & 2.2 & 3.7 & 274.9 & 43.7 \\
\hline 11.5 & 8.7 & 2.6 & 3.6 & 231.5 & 45.6 \\
\hline 11.2 & 9 & 3 & 5.26 & 159.4 & 43.6 \\
\hline 10.9 & 9.4 & 2.4 & 4.2 & 221.3 & 42.4 \\
\hline
\end{tabular}

Valores normales: calcio de 8.4-10.2 mg/DI, fósforo de 2.2-4.5 mg/DI y PTH de 31.4-90 pg/Ml.

tamizaje muy frecuentemente y principalmente en este tipo de institución, es muy fácil detectar esta patología en etapa temprana sin haber causado afección renal o tener alguna sintomatología, únicamente por parámetros alterados de laboratorio, por lo tanto hay que tenerla en mente.

La cirugía sigue siendo tratamiento curativo, con muy pocas complicaciones si es llevado por manos expertas; en nuestro estudio no hubo ninguna complicación, si es que llega a haber complicaciones podrían ser tan leves como una hipocalcemia transitoria o hipocalcemia persistente que ya requiera sustitución de calcio, incluso otra complicación aún más grave podría ser lesión de nervio laríngeo recurrente. Se debe considerar el uso de tratamiento médico únicamente a pacientes en quienes está contraindicada la cirugía por su estado de salud.

Para hacer un buen diagnóstico, es necesario tener estudios de sangre, para después hacer cirugía de mínima invasión con estudios de imagen de localización y PTH transoperatorio que baje $>50 \%$ para asegurarse que se ha resecado la glándula afectada.

\section{AGRADECIMIENTOS}

A la Facultad Mexicana de Medicina. Universidad La Salle México. 
La autora Estefanía Boyer agradece a Marco Sotomayor Sobrino por la asesoría brindada para el análisis estadístico del manuscrito.

\section{REFERENCIAS}

1. Yu N, Donnan PT, Murphy MJ, Leese GP. Epidemiology of primary hyperparathyroidism in Tayside, Scotland, UK. Clin Endocrinol (Oxf). 2009; 71 (4): 485-493.

2. Fraser WD. Hyperparathyroidism. Lancet. 2009; 374 (9684): 145-158.

3. Gasser RW. Clinical aspects of primary hyperparathyroidism: clinical manifestations, diagnosis, and therapy. Wien Med Wochenschr. 2013; 163 (17-18): 397-402.

4. Bilezikian JP, Bandeira L, Khan A, Cusano NE. Hyperparathyroidism. Lancet. 2018; 391 (10116): 168-178. doi: 10.1016/S01406736(17)31430-7.

5. Khan AA, Hanley DA, Rizzoli R, Bollerslev J, Young JE, Rejnmark L et al. Primary hyperparathyroidism: review and recommendations on evaluation, diagnosis, and management. A Canadian and international consensus. Osteoporos Int. 2017; 28 (1): 1-19.
6. Khan AA. Medical management of primary hyperparathyroidism. J Clin Densitom. 2013; 16 (1): 60-63.

7. Pallan S, Rahman MO, Khan AA. Diagnosis and management of primary hyperparathyroidism. BMJ. 2012; 344: e1013. doi: 10.1136/ bmj.e1013.

8. Granados Canseco E, Servín Torres E, Piscil Salazar MA, Velázquez García JA, Serrano Galeana I, Granados Canseco R et al. Experiencia quirúrgica en el hiperparatiroidismo primario en el Hospital de Especialidades del Centro Médico Nacional "La Raza". Cir Gen. 2012; 34 (4): 249-253.

9. Udelsman R, Akerstrom G, Biagini C, Duh QY, Miccoli P, Niederle B et al. The surgical management of asymptomatic primary hyperparathyroidism: proceedings of the Fourth International Workshop. J Clin Endocrinol Metab. 2014; 99 (10): 3595-3606.

10. Komaba H, Kakuta T, Fukagawa M. Management of secondary hyperparathyroidism: how and why? Clin Exp Nephrol. 2017; 21 (Suppl 1): 37-45.

11. Concistre A, Grillo A, La Torre G, Carretta R, Fabris B, Petramala L etal. Ambulatory blood pressure monitoring-derived short-term blood pressure variability in primary hyperparathyroidism. Endocrine. 2018; 60 (1): 129-137. 